\title{
¿Hay evidencia de ciclo político-económico en el empleo sectorial de México, 1998-2013?
}

\author{
Is there any evidence of political-business cycle in the sectorial \\ employment of Mexico, 1998-2013?
}

\author{
Pablo Mejía Reyes, Marlen R. Reyes Hernández * \\ y Liliana Rendón Rojas
}

Universidad Autónoma del Estado de México, México

Recibido el 2 de diciembre de 2014; aceptado el 3 de noviembre de 2015

Disponible en Internet el 23 de noviembre de 2016

\begin{abstract}
Resumen
El objetivo de este documento es determinar si existe evidencia de ciclo político-económico en el empleo sectorial de México durante el periodo 1998-2013. A partir de la estimación de modelos de panel, y controlando por los efectos de los principales determinantes del empleo, se encontró que no existe evidencia acorde con las predicciones del modelo teórico oportunista, es decir, si bien el empleo muestra un crecimiento antes y durante las elecciones, este resultado no es estadísticamente significativo ni robusto. Además, no se presenta la contracción del empleo posterior a los comicios o al cambio de poder. No obstante, se encuentra un efecto positivo de la producción y uno negativo de los salarios reales sobre el empleo.

(C) 2016 Universidad Nacional Autónoma de México, Facultad de Contaduría y Administración. Este es un artículo Open Access bajo la licencia CC BY-NC-ND (http://creativecommons.org/licenses/by-nc-nd/ $4.0 /)$.
\end{abstract}

Códigos JEL: D72; E32; E24; E61

Palabras clave: Ciclo político-económico; Empleo; Salarios; Producción

\footnotetext{
* Autor para correspondencia.

Correo electrónico: mar.rey2010@ hotmail.com (M.R. Reyes Hernández).

La revisión por pares es responsabilidad de la Universidad Nacional Autónoma de México.
} 


\begin{abstract}
This paper looks for evidence of political-business cycles associated to the presidential elections in the Mexican sectorial employment over the period 1998-2013. By estimating panel data models, and controlling for the effects of the major determinants of employment, no evidence consistent with the predictions of the theoretical opportunistic model is found, i.e. whereas employment shows an expansion before and during the elections periods, the estimates are neither statistically significant nor robust. Furthermore, employment does not experience contractions after the elections or the office taking periods. Notwithstanding, the evidence suggests that employment is positively and negatively affected by output and real wages, respectively.

(C) 2016 Universidad Nacional Autónoma de México, Facultad de Contaduría y Administración. This is an open access article under the CC BY-NC-ND license (http://creativecommons.org/licenses/by-nc-nd/4.0/).
\end{abstract}

JEL classification: D72; E32; E24; E61

Keywords: Political business cycle; Employment; Wages; Production

\title{
Introducción
}

La teoría del ciclo político-económico (CPE) estudia, en general, los efectos de las decisiones de política económica, condicionadas por eventos políticos, sobre el comportamiento de corto plazo de la economía. Su predicción más conocida es que el calendario electoral puede sumarse a los factores que explican las fluctuaciones cíclicas que experimentan las economías de mercado (Larraín y Assael, 1997). En particular, según Nordhaus (1975), pionero en el análisis moderno del tema, los gobiernos democráticos, independientemente de su ideología, buscan reelegirse o que su partido se mantenga en el poder. Para ello, hacen uso de los diferentes instrumentos de política económica a su alcance con el fin de generar resultados económicos de corto plazo favorables (como crecimiento de la producción y el empleo) que los hagan ver como gestores efectivos ante los electores, con lo que buscan inducir el voto de estos en su favor. De esta forma, se articulan medidas expansivas antes de las elecciones y, una vez celebradas, se aplican medidas estabilizadoras, buscando corregir las consecuencias negativas (incrementos de la inflación y desequilibrios fiscales, por ejemplo) de la política macroeconómica preelectoral. El patrón resultante de expansión de la actividad económica seguida de su contracción se denomina ciclo político-económico.

La investigación empírica sobre el tema, por otra parte, se inició en las economías desarrolladas a mediados de la década de los años setenta con el mismo trabajo de Nordhaus (1975), quien reporta evidencia de un patrón acorde al CPE en las tasas de inflación y de desempleo para los casos de Alemania, Nueva Zelanda y Estados Unidos durante el periodo de 1947 a 1972. A partir de entonces, el tema ha recibido mucha atención en la literatura, aunque no se ha establecido un claro consenso. Por ejemplo, Grier (1987) y Milani (2010) reportan evidencia de CPE en Estados Unidos durante los periodos 1961-1980 y 1966-2006, respectivamente, mientras que Berger y Woitek (1997) no hallan resultados acordes con el ciclo político oportunista en la producción, en los precios y en el desempleo de Alemania entre 1950 y $1989^{1}$.

\footnotetext{
${ }^{1}$ El tema también ha sido objeto de análisis en diferentes países en desarrollo. En particular, varios estudios han presentado evidencia que apoya la hipótesis de CPE para los países de América Latina, aunque también en este caso los resultados no han sido definitivos. Para mayores detalles, véanse los estudios de Remmer (1993), Larraín y Assael (1995) y Borsani (2003), entre otros.
} 
En el caso de México, usualmente queda la sensación de que las autoridades manipulan la política económica con el fin de mejorar temporalmente el desempeño de la economía como una forma de ganar la simpatía de los electores durante las elecciones presidenciales celebradas cada 6 años. De hecho, varios estudios han documentado estas prácticas para diferentes periodos y variables. Específicamente, los resultados de Magaloni (2000), Gámez (2004) y Cervantes, López y García (2014) muestran un comportamiento del PIB acorde a lo establecido en la teoría del $\mathrm{CPE}^{2}$. En particular, Magaloni (2000) encuentra tasas de crecimiento positivas y más altas antes de las elecciones explicadas por la formulación de políticas monetarias expansivas durante el periodo 1970-1982. Por su parte, Gámez (2004) sostiene que durante el periodo 1981-2004 se presentan tasas de crecimiento negativas después de celebrados los comicios. Por el contrario, Flores (2007), incorporando el PIB de Estados Unidos como variable de control en su modelo, no reporta evidencia de incrementos en las tasas de crecimiento de la economía antes de las elecciones durante el periodo 1983-2005, lo que atribuye al cambio hacia un enfoque más eficiente en el manejo de la política económica por parte de las nuevas administraciones públicas. Un análisis más completo es elaborado por Cervantes et al. (2014), quienes definen 2 subperiodos de estudio (1960-1983 y 1984-2011) y utilizan modelos de regresión lineal y vectores autorregresivos para analizar los efectos de variables como gasto público, base monetaria e inflación, además de variables binarias para capturar el efecto de antes, durante y después de las elecciones sobre el PIB real. Sus resultados sugieren la presencia de CPE principalmente durante el primer lapso. Los autores explican que estos resultados pueden deberse a la autonomía del Banco de México, que limita parcialmente la aplicación de políticas monetarias discrecionales, y a la apertura comercial, que hace más difícil influir sobre el ciclo económico que en una economía cerrada.

Por otro lado, la evidencia empírica relacionada con el desempleo no es consistente con las implicaciones de la teoría del CPE y su comportamiento puede explicarse por las políticas de estabilización seguidas en México durante el periodo 1983-1998, de acuerdo con Magaloni (2000), resultado validado también por Flores (2007) para el periodo 1983-2005.

Por último, un par de estudios pueden ser de interés especial para este trabajo porque analizan la dinámica de los sectores productivos ${ }^{3}$. En primer lugar, Morales (2007) estudia la relación entre el ciclo político y la producción de las 49 ramas del sector manufacturero durante el periodo 1994-2005 y encuentra que 18 de ellas tuvieron un comportamiento parcialmente acorde al CPE: 4 sufrieron una expansión antes del cambio de poder y 14 presentaron una contracción después de este evento. Gámez (2010), a su vez, analiza el PIB por sector de origen para el periodo que va de 1962 a 2007 y también identifica evidencia parcial de CPE al señalar que los efectos principales no se manifiestan antes de las elecciones sino después de ellas, en especial después del cambio de poder presidencial. Más aun, sostiene que la contracción es más pronunciada en los sectores con mayor vinculación al mercado interno, como el comercio, ciertos servicios y, especialmente, la industria de la construcción.

En general, resulta interesante observar que la mayor parte de los análisis sobre CPE que se han llevado a cabo para México se refieran a los efectos de distintas políticas o a la dinámica de diferentes medidas de producción y que muy pocos hagan alusión explícita al empleo. No obstante, resulta intuitivamente atractiva la idea de que los efectos de las conductas oportunistas

\footnotetext{
${ }^{2}$ Magaloni (2000) y Gámez (2004) estiman modelos autorregresivos para captar la dinámica de la serie analizada y variables binarias para estimar el efecto de las elecciones. Algunos de estos estudios, y otros, consideran otras variables resultado, como la inflación o la demanda agregada. Para mayores detalles, véase Gámez (2004, 2012), Flores (2007) y Cervantes et al. (2014).

${ }^{3}$ En ambos casos se estiman modelos autorregresivos para cada serie.
} 
de los gobernantes sobre el empleo les puede ganar más votos ya que los ciudadanos percibirían de manera directa, con un puesto de trabajo y el ingreso que de él se deriva, los supuestos efectos benéficos de las políticas oportunistas de los gobiernos en turno. Así pues, el objetivo de este artículo es establecer si el empleo sectorial de México muestra una conducta cíclica asociada a las elecciones presidenciales y al cambio de poder, es decir, si experimenta un incremento antes y durante estos procesos y una declinación posterior. Para abordar el tema, se modela el crecimiento del empleo formal total de los 9 sectores de actividad económica durante el período 1998-2013 ${ }^{4}$, que incluye 3 elecciones presidenciales, mediante la estimación de modelos de panel que incorporan variables electorales (ficticias) y de control. Las primeras capturan el efecto de los comicios y se especifican de 2 formas distintas para tomar en cuenta el efecto directo de las elecciones (usualmente celebradas a principios de julio) y el papel del periodo transcurrido entre estas y la toma de poder (el 1 de diciembre). Por su parte, las variables de control se refieren al salario real, como medida principal del costo del empleo, y a la producción, como medida de la demanda efectiva que enfrentan las empresas.

El resto de este artículo se organiza de la siguiente manera. En la primera sección se exponen brevemente la teoría del CPE y los efectos esperados de los determinantes más importantes del empleo, salario real y producción, con base en los planteamientos de la nueva economía keynesiana. En la segunda sección se desarrolla la metodología de los modelos econométricos para datos de panel y se especifican los modelos a estimar. Los resultados obtenidos son reportados y discutidos en la tercera sección. Finalmente, en la última, se establecen las conclusiones.

\section{Consideraciones teóricas}

\section{La teoría del ciclo político-económico}

La teoría del CPE analiza la interacción entre los sistemas político y económico, y parte del supuesto de que a los votantes les importa el desempeño de la economía y a los gobernantes les interesa mantenerse en el poder. Los modelos que tratan de explicar el CPE se pueden clasificar de acuerdo a 2 criterios. Según el objetivo que persigue el partido en el poder pueden ser de tipo oportunista o partidario (también llamado ideológico). Los primeros suponen que, independientemente de su ideología, el único interés de los partidos es alcanzar o mantenerse en el poder, mientras que los otros parten de la idea de que lo que buscan es implementar un programa de gobierno específico basado en sus principios ideológicos ${ }^{5}$. Por otro lado, en relación con las expectativas que se asumen para los agentes, los modelos pueden ser de expectativas adaptativas o racionales. Los primeros corresponden a modelos tradicionales en los que los gobiernos pueden influir en los escenarios macroeconómicos de manera sistemática y predecible y donde la «memoria corta» del electorado explica la aparición del CPE. En cambio, en los modelos con expectativas racionales la información asimétrica entre elector y candidato explica su aparición (Nordhaus, 1975; Hibbs, 1977; Alesina, Roubini y Cohen, 1999) ${ }^{6}$.

\footnotetext{
${ }^{4}$ Aunque el análisis se centra solamente en el formal, vale la pena señalar que este es el indicador más comúnmente usado para medir el empleo, además de que no existe información oficial del empleo total sino a partir de 2005 , lo que implicaría reducir el análisis del CPE solamente a la elección de 2012.

${ }^{5}$ En general, los modelos partidarios distinguen 2 grandes tendencias ideológicas: los denominados partidos de izquierda, que dan prioridad al crecimiento del empleo, aun a costa de mayores niveles de inflación, y los de derecha, que tienen preferencias contrarias (Hibbs, 1977).

${ }^{6}$ Los estudios que incorporan expectativas racionales aparecen a mediados de los ochenta. En estos modelos los individuos forman sus expectativas sobre la eficiencia del candidato y están al tanto de los incentivos de las autoridades,
} 
La experiencia de diversos países durante las últimas décadas, entre ellos México, nos inclinan hacia una interpretación basada en los modelos que asumen conductas oportunistas de los gobernantes y expectativas no racionales ${ }^{7}$, de acuerdo a los planteamientos de Nordhaus (1975) y Mac Rae (1977).

Los supuestos fundamentales que adopta Nordhaus (1975) pueden sintetizarse en 2 puntos: 1) los votantes eligen a sus representantes políticos sobre la base de los resultados económicos recientes, es decir, tienen una memoria corta del pasado, y 2) los políticos están restringidos por la existencia de una estructura económica dada, que puede caracterizarse, en términos generales, por medio de una curva de Phillips ampliada con «expectativas adaptativas» (Villasuso, 2000). Bajo estos supuestos se puede derivar una orientación concreta en la formulación de la política económica: los gobiernos siguen diferentes reglas de política económica en función de la proximidad o lejanía de las elecciones, de modo que se articulan medidas expansivas antes de las mismas y, una vez celebradas, se aplican medidas restrictivas que buscan corregir las consecuencias negativas de la política macroeconómica preelectoral (Molina, 2001). Como consecuencia, en un CPE típico, la economía vive una expansión de la actividad económica y del empleo antes de cada elección, así como un posible aumento de la tasa de inflación alrededor de la fecha de elecciones. Por el contrario, se espera una reducción postelectoral de la inflación resultante de las políticas restrictivas que también originan una desaceleración o caída de la producción y del empleo ${ }^{8}$.

\section{Determinantes del empleo: salario real y producción}

Existen 2 teorías principales que explican la dinámica del mercado de trabajo: la neoclásica y la keynesiana. La primera asume que el trabajo puede considerarse como cualquier otra mercancía, que responde a las fuerzas de la oferta y la demanda. En ese sentido, la oferta de trabajo es una función directa del salario real, de manera que a medida que este aumenta se eleva el costo de oportunidad del ocio, por lo que la maximización de utilidad del trabajador lo llevará a elevar su disposición a trabajar, y viceversa. Por su parte, la demanda de trabajo responde a la maximización de ganancias del productor, lo que implica que demandará el insumo trabajo hasta el punto en el que el salario real iguale a su productividad marginal. Dada la existencia de rendimientos decrecientes en el uso del trabajo, esa relación tendrá una pendiente negativa a medida que aumenta el nivel de empleo. La flexibilidad del salario garantiza que se alcanzará el equilibrio de mercado en el punto donde coincidan las decisiones de oferta y demanda de trabajo de los participantes del mercado (Romer, 2011).

pero no tienen toda la información con que cuentan estas sobre su capacidad y motivaciones reales (información asimétrica), por lo que los electores pueden ser engañados, aunque no de manera sistemática. Como consecuencia, el CPE es más corto y no regular (Borsani, 2003).

7 Los países que han integrado procesos democráticos a su vida política de manera reciente, debido a que vienen de gobiernos dictatoriales o a que la competencia real entre partidos no existía, se caracterizan por la existencia de instituciones débiles, con poca capacidad regulatoria, y electores con baja educación política, que los hace susceptibles a la manipulación (Rogoff, 1990). Además, en el caso de México, no existe evidencia de que la alternancia política haya estado asociada a diferentes visiones sobre el manejo de la economía. De hecho, el programa económico del partido gobernante en el año 2000 (Partido Revolucionario Institucional [PRI]), cuando se da la primera alternancia, era esencialmente el mismo que el del partido que lo sucedió (Partido Acción Nacional [PAN]). Análogamente, la estrategias perseguidas por el PAN hacia el año 2012 fueron similares a las que ha adoptado el PRI a partir de entonces (Camacho, 2014).

${ }^{8}$ También, es probable que el gobernante reduzca los impuestos o aumente el gasto público, o ambas cosas a la vez, en el periodo preelectoral, y es altamente improbable que se efectúe un ajuste económico durante ese período. Los ajustes y alzas de impuestos, cuando ocurren, tienden a suceder después de las elecciones (Larraín y Assael, 1997). 
Una perspectiva diferente sobre el funcionamiento del mercado de trabajo la ofrece la teoría keynesiana en sus diferentes vertientes. En particular, la llamada nueva economía keynesiana sostiene que el nivel de empleo depende de la demanda efectiva y que el desempleo se puede explicar por la presencia de salarios superiores a los de equilibrio, que reducen la demanda en relación con la oferta y que son esencialmente rígidos ${ }^{9}$. En esta visión, que contrasta con la neoclásica, los salarios reales determinan el esfuerzo y la productividad de los trabajadores, quienes son incentivados a ser más eficientes y productivos con un pago más alto denominado salario de eficiencia, concepto que trata de enfatizar la causalidad del salario hacia la productividad (Malcomson, 1981; Gordon, 1990 $)^{10}$. En ese sentido, un incremento en el salario de eficiencia podría desplazar la curva de demanda de empleo, provocando un incremento en este, lo que definiría una relación positiva entre estas variables. Alternativamente, el salario pactado entre trabajadores y empresarios podría exceder al de mercado si los primeros (internos) tuvieran cierto poder para determinarlo derivado de los costos de contratación y despido o de capacitación asociados a la alta rotación de empleados. Con el fin de eliminar o reducir esos costos, los empresarios tendrían incentivos para pagar un salario superior al de equilibrio aun cuando hubiera trabajadores desempleados (externos) dispuestos a aceptar un salario inferior (Lindbeck y Snower, 1991). Bajo estas consideraciones, se mantiene la relación inversa convencional entre las variaciones del salario y del empleo. En cualquiera de los 2 casos, sin embargo, se esperaría que la rigidez salarial se tradujera en un efecto modesto sobre el empleo y que su determinante más importante fuera la demanda efectiva, usualmente aproximada por alguna medida de producción.

\section{Metodología econométrica}

Para medir el efecto de las variables descritas en la sección anterior sobre el crecimiento del empleo se utiliza un modelo para datos de panel ${ }^{11}$. Como es bien sabido, estos modelos detectan y son más eficientes para medir los efectos de cambio que no se observan en otras técnicas econométricas, pues al combinar las series de tiempo con las observaciones de corte transversal se proporciona mayor información y más grados de libertad, así como menor colinealidad entre variables, mayor eficiencia y menos posibilidades de sesgo en la estimación. Además, al combinar muestras aleatorias extraídas de la misma población, pero en distintos puntos del tiempo, se obtienen estimadores más precisos y estadísticos con mayor potencia de prueba (Wooldridge, 2009). Más aún, la aplicación de esta metodología permite analizar 2 aspectos cuando se trabaja con datos heterogéneos: a) los efectos individuales específicos, y b) los efectos temporales (Mayorga y Muñoz, 2000).

\footnotetext{
9 Véase Romer (2011) para una exposición formal.

10 Para justificar la existencia de salarios superiores a los de equilibrio, aceptados desde luego por lo empresarios, se han planteado modelos de diferente índole sobre la base de 4 argumentos. Primero, aumentan el esfuerzo de los trabajadores y, por lo tanto, afectan positivamente su productividad. Segundo, no solo contribuyen a mejorar las capacidades de los trabajadores, sino que atraen a los más capacitados y, por lo tanto, aumentan su productividad media. Tercero, estimulan un sentimiento de lealtad en los trabajadores hacia la empresa e inducen un mayor esfuerzo. Por último, aunque elevan los costos laborales de la empresa, por otro lado disminuyen los costos de supervisión o monitoreo en que tendría que incurrir la empresa para garantizar un mínimo de productividad si pagara salarios bajos (Mankiw y Romer, 1991).

${ }^{11}$ Los datos de panel son observaciones repetidas de la misma serie de tiempo para un conjunto de unidades, ya que se tiene el mismo número de observaciones para cada unidad cruzada (Johnston y DiNardo, 1997). Es así como los modelos de panel son anchos, pero en general cortos (Greene, 1998).
} 
En esta investigación los modelos para datos de panel permiten incorporar en el análisis el hecho de que los sectores productivos son heterogéneos y al mismo tiempo posibilitan el control por factores específicos de cada uno de ellos asociados a la heterogeneidad no observable ${ }^{12}$.

Para estimar los efectos de las elecciones presidenciales sobre el crecimiento del empleo $(l)$ y determinar si existe evidencia de CPE, controlando por los efectos del crecimiento del salario real $(w)$ y del de la producción $(y)$, se formulan 2 modelos distintos definidos en las expresiones (1) y (2). El primero de ellos se plantea en los siguientes términos:

$$
l_{i t}=\alpha+\beta_{1} y_{i t}+\beta_{2} w_{i t}+\gamma_{a} E_{t}^{a}+\gamma_{d} E_{t}^{d}+u_{i t}
$$

donde las $E_{t}$ son variables dicótomas que representan las elecciones presidenciales y que toman el valor de 1 los 4 trimestres previos a la elección (superíndice $a$ ) y los 4 posteriores, incluido el trimestre de la elección (superíndice $d$ ) ${ }^{13}$, y 0 en cualquier otro periodo. Los parámetros a estimar y sus signos esperados son $\alpha \gtrless 0, \beta_{1}>0, \beta_{2}<0, \gamma_{a}>0$ y $\gamma_{d}<0$. Si los 2 últimos coeficientes cumplen estas condiciones y son estadísticamente significativos, la tasa de crecimiento media del empleo sectorial sería mayor antes de las elecciones y menor posteriormente.

En el modelo 2 se incluye la posibilidad de que el periodo transcurrido entre la elección y la toma de poder tenga efectos diferenciados sobre el empleo. Esta distinción es importante dado que ese intervalo de tiempo es de aproximadamente 5 meses y se caracteriza por un alto grado de inactividad gubernamental. Además, aunque el nuevo gobierno entra en funciones el 1 de diciembre, realmente puede aplicar medidas de política económica hasta el 1 de enero del siguiente año de acuerdo al calendario de ejecución del presupuesto de egresos y de la ley de ingresos (Centro de Estudios de las Finanzas Públicas, 2014). Para captar esta posibilidad se incorpora la variable dicótoma $E_{t}^{p}$, que toma el valor de 1 en el trimestre de la elección y en el trimestre posterior, de manera que el modelo 2 puede formularse como sigue:

$$
l_{i t}=\alpha+\beta_{1} y_{i t}+\beta_{2} w_{i t}+\gamma_{a} E_{t}^{a}+\gamma_{p} E_{t}^{p}+\gamma_{d} E_{t}^{d}+u_{i t}
$$

donde se espera que $\gamma_{p}<0$. $E_{t}^{a}$ se define del mismo modo, y $E_{t}^{d}$ toma un valor de 1 durante los 4 trimestres del primer año del siguiente gobierno, y 0 en cualquier otro caso.

En relación con la especificación del modelo para datos de panel, se debe mencionar que $\alpha$ se puede asociar al modelo de datos agrupados, el cual contiene un intercepto y un coeficiente pendiente común para todas las unidades; el modelo se estima por mínimos cuadrados ordinarios (MCO) siempre y cuando la cov $\left(l_{i t}, u_{i t}\right)=0$. Sin embargo, cuando este supuesto no se cumple, por algún error de especificación, se tendrán estimadores sesgados e inconsistentes. Para resolver el problema se puede plantear el modelo como uno de datos anidados con 2 opciones, de efectos fijos (EF) o de efectos aleatorios (EA).

En el caso de EF, la heterogeneidad no observable se incorpora en la ordenada al origen del modelo, alterando el valor esperado de la variable explicada o endógena. Se supone que aunque el intercepto puede diferir entre las unidades no varía con el tiempo, es decir, $\alpha=\alpha_{i}$. Entonces se tendrá una parte fija, constante a través del tiempo para cada individuo, y otra aleatoria que cumple los requisitos de $\mathrm{MCO}$, lo que permite obtener un coeficiente pendiente general de la

\footnotetext{
${ }^{12}$ Los modelos de series de tiempo y de corte transversal no tratan de controlar esta heterogeneidad, corriendo el riesgo de obtener estimaciones sesgadas.

${ }^{13}$ El trimestre de la elección se incluye en el periodo posterior a la elección debido a que esta se lleva a cabo los primeros días de julio y a que por ley las actividades proselitistas deben terminarse 3 días antes de la fecha electoral (Cámara de Diputados del Congreso de la Unión, 2014).
} 
regresión y un intercepto específico para cada unidad que recoge los elementos idiosincrásicos no observables que no dependen del tiempo ${ }^{14}$.

En cambio, en el modelo de EA las diferencias no observables se incorporan al término del error, y es la varianza del modelo la que se modifica, de manera que el término independiente $\alpha_{i}$ es una variable aleatoria. El valor del intercepto se expresa como $\alpha_{i}=\alpha+\varepsilon_{i}$, donde $\varepsilon_{i}$ es un término de error aleatorio con valor medio igual a cero y varianza $\sigma_{s}^{2}$. Entonces, delineamos el $\varepsilon_{i}$ $+u_{\mathrm{it}}=w_{\text {it }}$ como error compuesto, donde $\varepsilon_{i}$ es el componente de error de corte transversal y $u_{\mathrm{it}}$ es la combinación del componente de error de series de tiempo y de corte transversal, es decir, varía con los sujetos y en el tiempo, siendo las perturbaciones homoscedásticas. El método más apropiado para estimar estos modelos es por mínimos cuadrados generalizados (Greene, 1998).

En general, el modelo de EF es más apropiado cuando el panel contiene a todos los individuos de la muestra y el de EA cuando se tiene solo una muestra. Además, se debe establecer que el modelo de EA genera estimaciones más eficientes, pero el de EF se asocia a estimaciones más consistentes (Greene, 1998; Wooldridge, 2009; Montero, 2011).

Ahora bien, para determinar si debe emplearse un modelo de datos anidados (EA) o simplemente el modelo de datos agrupados, se utiliza la prueba tipo multiplicador de Lagrange propuesta por Breusch y Pagan (1980), la cual evalúa la hipótesis nula de que $\sigma_{s}^{2}=0$. Si la prueba se rechaza, es preferible usar el método de EA que el modelo agrupado. Por otro lado, se puede emplear una prueba $F$ estándar que evalúa la significancia estadística de los coeficientes de las variables binarias individuales introducidas para captar los EF: si la hipótesis nula no se puede rechazar, se opta por el modelo de datos agrupados; en caso contrario, se prefiere el modelo de EF.

Por su parte, para decidir entre un modelo de EF y de EA se utiliza la prueba de Hausman $(1978)^{15}$, la cual contrasta las estimaciones de ambos modelos. Si se encuentran diferencias significativas, se rechaza la hipótesis nula de igualdad debido a que, asumiendo una especificación razonablemente aceptable, seguiría existiendo correlación entre el término de error y los regresores; en tal caso, se opta por el modelo de EF que genera estimaciones consistentes. Si, por el contrario, las estimaciones son significativamente iguales, se elige el modelo de EA que ofrece estimaciones más eficientes (Greene, 1998; Aparicio y Márquez, 2005; Wooldridge, 2009).

\section{Resultados}

\section{Información estadística y evidencia preliminar del ciclo político-económico}

Los modelos especificados en la sección anterior se estimaron para el periodo 1998-2013, el cual, aunque estuvo determinado esencialmente por la disponibilidad de información estadística, incluye 3 elecciones presidenciales. En particular, las series de producción utilizadas corresponden a los componentes sectoriales del Indicador Global de la Actividad Económica (IGAE) publicado por el Instituto Nacional de Estadística y Geografía (INEGI), mientras que las variables de empleo y salarios nominales se refieren al número de trabajadores afiliados al Instituto Mexicano del

\footnotetext{
${ }^{14}$ La estimación se puede llevar a cabo por MCO introduciendo una variable binaria por individuo, aunque en la práctica es más común su estimación tomando las variables como diferencias con respecto a su media.

15 Es una prueba clásica de robustez (robustness) frente a eficiencia. Este tipo de pruebas plantean 2 estimadores para un mismo conjunto de parámetros: uno robusto, $\theta_{R}$, consistente tanto bajo la hipótesis nula $H_{0}$ como bajo la alternativa $H_{1}$, y otro eficiente, $\theta_{E}$, pero solo bajo la hipótesis nula $H_{0}$. Si una vez calculados ambos la diferencia observada entre los 2 estimadores $\left(\theta_{R}-\theta_{E}\right)$ es escasa, se toma evidencia a favor de la hipótesis nula (Mahía, 2000).
} 


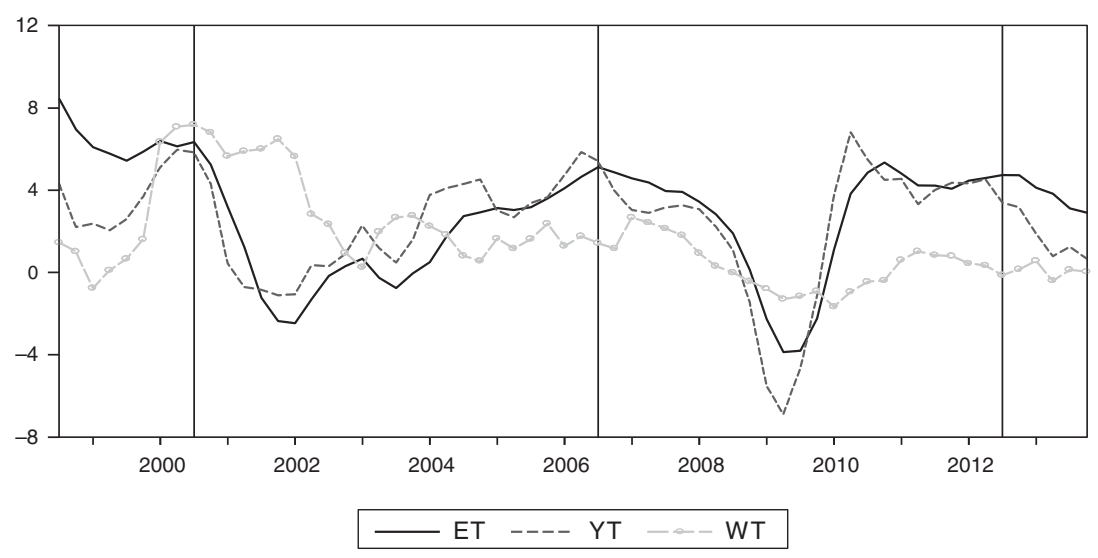

Figura 1. México: empleo, producción y salarios, 1998-2013 (tasas de crecimiento anualizadas). Fuente: elaboración propia con base en datos del INEGI y del IMSS.

Seguro Social (IMSS) y a las cotizaciones con que estos son registrados para cada sector ${ }^{16}$. Los datos originales se publican con una periodicidad mensual, pero se pasaron a series trimestrales, tomando el promedio de los datos de los meses correspondientes, con el objetivo de reducir su volatilidad y hacer factible su modelaje ${ }^{17}$. Por su parte, los salarios nominales fueron deflactados con el índice de precios implícito del PIB de cada sector con el objeto de tener una medida más específica del costo real que pagan los empresarios.

Se obtuvieron las tasas de crecimiento anualizadas de las 3 variables tanto para la economía nacional como para los diferentes sectores con el objeto de tener una transformación estacionaria que posibilitara la aplicación de métodos de estimación convencionales. Con fines ilustrativos, en la figura 1 se muestra la evolución de las tasas de crecimiento del empleo, $L T$, la producción, $Y T$, y los salarios reales, $W T$, a nivel nacional, así como las fechas en las que se llevaron a cabo las elecciones presidenciales, indicadas con líneas verticales. En principio, se puede ver que las variaciones en las tasas del empleo se han visto acompañadas de movimientos similares en las de la producción y que las altas tasas de crecimiento del empleo han correspondido a menores tasas de los salarios reales, y viceversa.

Además, se puede apreciar que el comportamiento de las tasas de crecimiento del empleo es parcialmente consistente con la hipótesis del CPE debido a que se presentan tasas de crecimiento mayores antes de las elecciones, especialmente de las de 2000 y 2006, y caídas posteriores a las 3 elecciones celebradas en el periodo. No obstante, se debe subrayar que la contracción que siguió a las elecciones de 2000 se puede atribuir a la recesión iniciada a finales de ese año y a las políticas restrictivas empleadas para superarla (Mejía y Erquizio, 2012), y que la de 2013 se debió en gran parte a la desaceleración de la economía estadounidense, pero también al extraordinario recorte del gasto público (SHCP, 2013). La dinámica del empleo en torno a estas elecciones evidencia

\footnotetext{
${ }^{16}$ El IGAE se publica desde enero de 1993, y los datos de empleo y salarios, desde julio de 1997. Es importante aclarar que este indicador de empleo se refiere solamente a los trabajadores del sector productivo formal, dejando fuera a los trabajadores del sector público y a los del sector informal. Los datos fueron tomados de la sitios de internet de estas instituciones: <www.inegi.org.mx> y <www.imss.gob.mx>, respectivamente.

${ }^{17}$ Las series de empleo y salarios se desestacionalizaron usando el método X-12 ARIMA de EViews 7.0; las series de producción se encontraron desestacionalizadas en la fuente.
} 
la necesidad de tomar en cuenta el efecto de factores económicos asociados al ciclo económico para aislar, en la medida de lo posible, los efectos atribuibles a las elecciones.

Los paneles de la figura 2, por su parte, muestran las tasas de crecimiento de las mismas variables para los 9 sectores analizados en este documento, organizados de la siguiente forma: a) agricultura, ganadería, silvicultura, caza y pesca (AGS); b) Industrias extractivas (IEXT); c) industrias de la transformación (ITRANS); d) construcción (CONS); e) industria eléctrica y suministro de agua potable (IEAG); f) comercio (COMER); g) transportes y comunicaciones (TRANYCOM); h) servicios para empresas, personas y hogar (SERV), e i) servicios sociales y comunales
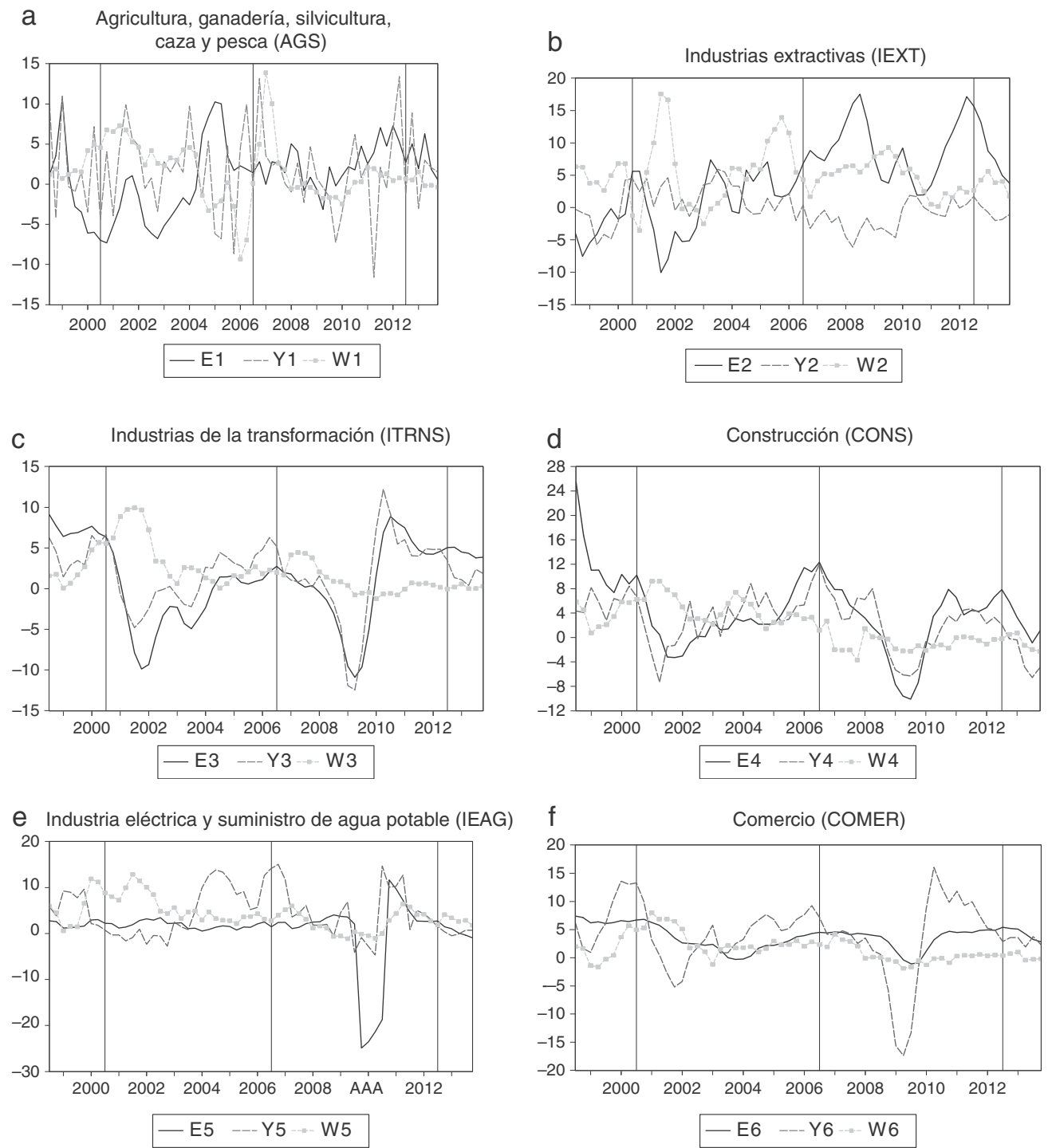

Figura 2. México: empleo, producción y salarios por sectores, 1998-2013 (tasas de crecimiento anualizadas).

Fuente: elaboración propia con base en datos del INEGI y del IMSS. 


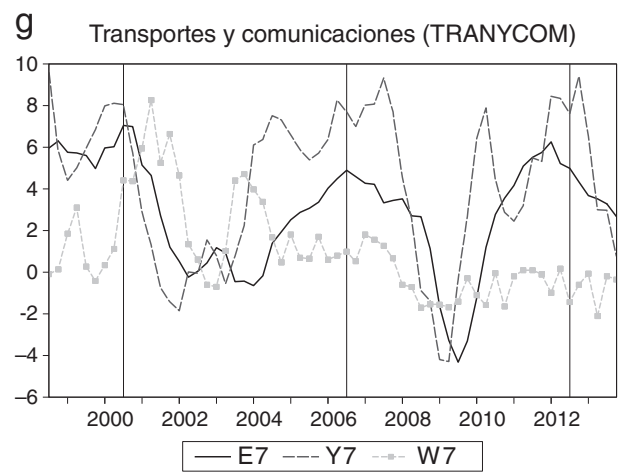

$\mathrm{h}$ Servicios para empresas, personas y hogar (SERV)
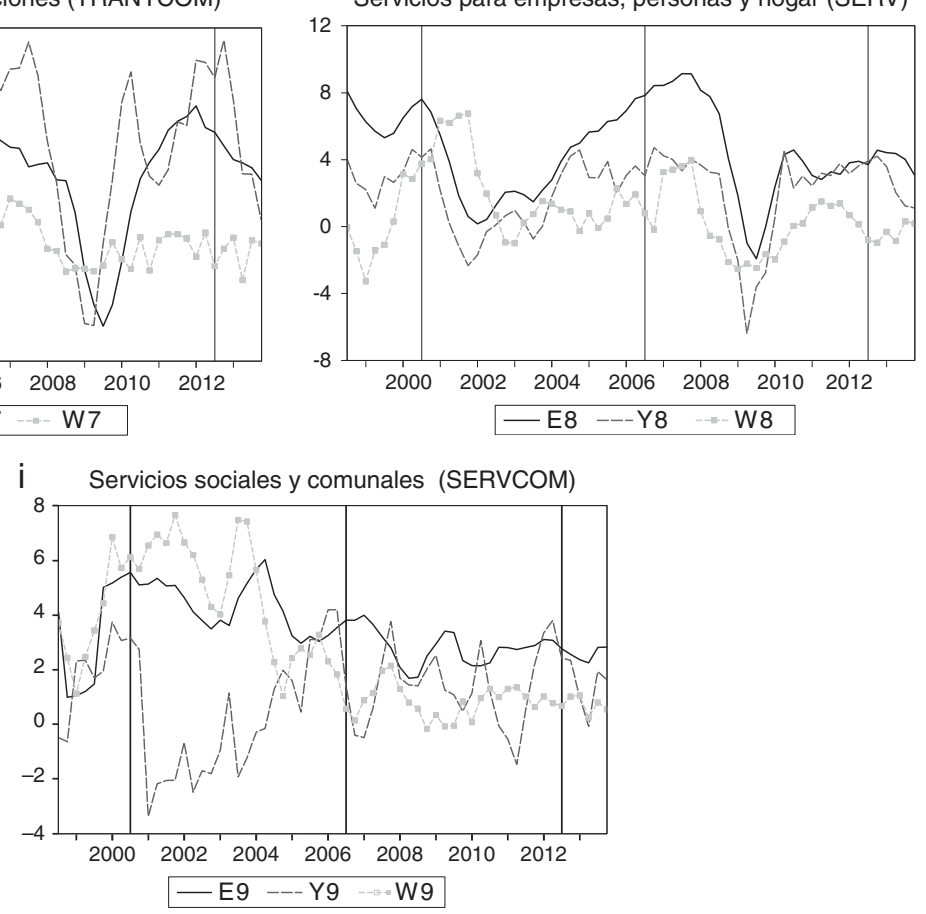

Figura 2 (Continuación).

(SERVCOM). Las tasas de crecimiento del empleo, la producción y los salarios reales del $i$-ésimo sector se denotan como $L i, Y i$ y $W i$ (para $i=1,2, \ldots, 9$ ), respectivamente.

Como se puede apreciar, el comportamiento de las tasas de crecimiento del empleo y su relación con el crecimiento de la producción y los salarios reales difieren de manera sustancial entre los distintos sectores. En algunos casos, como en ITRANS, CONS y SERV, se presenta un patrón similar al nacional, pero en otros las diferencias son notables, como en AGS e IEXT. Análogamente, se pueden identificar desempeños compatibles, en principio, con el CPE en los primeros 3 sectores citados, aunque no en los otros.

La información de la tabla 1 también permite identificar evidencia preliminar de CPE: se muestran las tasas de crecimiento promedio del empleo de los 9 sectores de la economía durante los periodos anterior y posterior a las 3 elecciones presidenciales, de acuerdo con el modelo definido en la ecuación 1, así como el promedio del periodo total. En primer lugar, se puede ver que el empleo presenta tasas de crecimiento muy diferentes para los distintos sectores durante el periodo completo. En segundo lugar, en el periodo preelectoral se observan tasas más elevadas que el promedio de todo el periodo, especialmente antes de las elecciones de 2000 y 2012, lo que podría ser consistente con el CPE. Sin embargo, las tasas de crecimiento promedio del periodo postelectoral también fueron mayores que las del periodo completo en la mayoría de los casos, lo que es incompatible con las predicciones de la teoría del CPE. En la tabla 2 se presentan las tasas de crecimiento de los sectores durante el periodo previo, de transición y posterior a las 3 elecciones, de acuerdo al modelo 2. En este caso también se observan tasas de crecimiento 
Tabla 1

Tasas de crecimiento promedio del empleo por sectores durante los periodos previo (CPEA) y posterior (CPED) a las elecciones

\begin{tabular}{|c|c|c|c|c|c|c|c|}
\hline \multirow{2}{*}{$\begin{array}{l}\text { Periodo electoral } \\
\text { Sector }\end{array}$} & 2000 & 2006 & 2012 & 2000 & 2006 & 2012 & \multirow[t]{2}{*}{$\begin{array}{l}\text { Promedio } \\
1998-2013\end{array}$} \\
\hline & \multicolumn{3}{|c|}{ CPEA } & \multicolumn{3}{|c|}{ CPED } & \\
\hline AGS & -4.6 & 2.3 & 6.1 & -5.6 & 1.8 & 4.0 & 1.0 \\
\hline IEXT & -1.2 & 2.4 & 13.1 & 2.0 & 7.7 & 11.3 & 4.6 \\
\hline ITRANS & 7.2 & 1.2 & 4.5 & 2.1 & 1.8 & 4.8 & 0.8 \\
\hline CONS & 8.8 & 9.3 & 5.1 & 4.9 & 9.4 & 4.6 & 3.4 \\
\hline IEAG & 2.3 & 1.8 & 3.2 & 1.8 & 1.9 & 1.4 & 0.8 \\
\hline COMER & 6.3 & 3.7 & 4.8 & 6.4 & 4.5 & 5.0 & 3.6 \\
\hline TRANYCOM & 5.7 & 3.7 & 5.7 & 6.0 & 4.5 & 4.1 & 2.8 \\
\hline SERV & 6.1 & 6.8 & 3.5 & 5.9 & 8.4 & 4.3 & 4.4 \\
\hline SERVCOM & 4.3 & 3.3 & 3.0 & 5.3 & 3.8 & 2.5 & 3.5 \\
\hline TOTAL & 6.0 & 3.9 & 4.3 & 4.0 & 4.7 & 4.4 & 2.7 \\
\hline
\end{tabular}

En negrita, las tasas más altas.

Fuente: elaboración propia.

mayores al promedio del periodo completo, tanto en el periodo de transición como en el posterior, contrario a lo sugerido por el modelo teórico.

Esta evidencia preliminar, no obstante, debe evaluarse formalmente, tarea que se desarrolla en la siguiente sección.

\section{Estimación}

La estimación de los modelos 1 y 2 definidos en la sección 2 se realizó con métodos para datos agrupados y de panel, con EF y EA, tomando como base las tasas anualizadas de crecimiento de las variables de empleo, producción y salarios reales; los resultados se presentan en la tabla $3^{18}$. En primer lugar, se puede mencionar que los resultados de las pruebas $F$ y de Breusch y Pagan (1980), explicadas previamente, sugieren que los modelos para datos de panel son más adecuadas, dado que los valores $p$ correspondientes, que aparecen al pie del tabla 3, sugieren que la hipótesis nula de datos agrupados se rechaza al $1 \%$. Por su parte, los valores $p$ correspondientes a la prueba de Hausman (1978) sugieren que el modelo de EA es más apropiado (al 5\% de significancia) que el modelo de EF. Adicionalmente, los valores $p$ del estadístico $F$ de la prueba de Wooldridge (2009) ${ }^{19}$ indican la presencia de autocorrelación y heteroscedasticidad. Para corregir ambos problemas, la estimación se llevó a cabo por Mínimos Cuadrados Generalizados Factibles (MCGF) y con

\footnotetext{
${ }_{18}$ Como es bien sabido, los métodos convencionales pueden aplicarse si las series son estacionarias. Aunque algunas tasas de crecimiento muestran un comportamiento que parece no estacionario, como se observa en los paneles de la figura 2 , la prueba de Im, Pesaran y Shin (2003), que incorpora un número diferente de rezagos en las ecuaciones del modelo de panel, sugiere que las series son estacionarias cuando se consideran en conjunto: los valores $p$ del estadístico $Z_{i b a r}$ son iguales a 0.0010, 0.0000 y 0.0000 para el empleo, el salario y la producción, respectivamente, por lo que la hipótesis nula de raíz unitaria se rechaza al $1 \%$ de significancia. Estos resultados y las pruebas convencionales de estacionariedad para las series individuales están disponibles bajo solicitud expresa.

${ }^{19}$ Esta prueba utiliza los residuos de una regresión en primeras diferencias, observando que si $u_{i t}$ no está serialmente correlacionada, entonces la correlación entre los errores $u_{i t}$ diferenciados para los periodos $t$ y $t-1$ deberá ser igual a -0.5 . Drukker (2003) presenta evidencias de que esta prueba tiene propiedades robustas para tamaños de muestra razonable. En este caso, el valor $p$ correspondiente permite rechazar la hipótesis nula al $1 \%$ en ambos modelos.
} 
Tabla 2

Tasas de crecimiento promedio del empleo por sectores durante los periodos previo (CPEA), de transición (CPEP) y posterior (CPED) a las elecciones

\begin{tabular}{|c|c|c|c|c|c|c|c|c|c|c|}
\hline \multirow{2}{*}{$\begin{array}{l}\text { Periodo electoral } \\
\text { Sector }\end{array}$} & $\begin{array}{l}1999 \\
\text { t3-2000 t2 }\end{array}$ & $\begin{array}{l}2005 \\
\text { t3-2006 t2 }\end{array}$ & $\begin{array}{l}2011 \\
\text { t3-2012 t2 }\end{array}$ & $\begin{array}{l}2000 \\
t 3-2000 t 4\end{array}$ & $\begin{array}{l}2006 \\
\text { t3-2006 t4 }\end{array}$ & $\begin{array}{l}2012 \\
\text { t3-2012 t4 }\end{array}$ & $\begin{array}{l}2001 \\
\mathrm{t} 1-2001 \mathrm{t} 4\end{array}$ & $\begin{array}{l}2007 \\
\mathrm{t} 1-2007 \mathrm{t} 4\end{array}$ & $\begin{array}{l}2013 \\
\mathrm{t} 1-2013 \mathrm{t} 4\end{array}$ & \multirow{2}{*}{$\begin{array}{l}\text { 1998-2013 } \\
\text { Total del } \\
\text { periodo }\end{array}$} \\
\hline & \multicolumn{3}{|c|}{ CPEA } & \multicolumn{3}{|c|}{ CPEP } & \multicolumn{3}{|c|}{ CPED } & \\
\hline AGS & -4.6 & 2.3 & 6.1 & -7.1 & 2.1 & 3.9 & -1.6 & 1.7 & 2.7 & 1.0 \\
\hline IEXT & -1.2 & 2.4 & 13.1 & 5.6 & 7.8 & 14.5 & -5.3 & 8.7 & 6.2 & 4.6 \\
\hline ITRANS & 7.2 & 1.2 & 4.5 & 5.4 & 2.4 & 5.1 & -5.1 & 0.8 & 4.1 & 0.8 \\
\hline CONS & 8.8 & 9.3 & 5.1 & 8.5 & 11.0 & 6.8 & -1.1 & 6.3 & 1.2 & 3.4 \\
\hline IEAG & 2.3 & 1.8 & 3.2 & 2.2 & 2.0 & 2.1 & 1.8 & 1.8 & 0.0 & 0.8 \\
\hline COMER & 6.3 & 3.7 & 4.8 & 6.8 & 4.5 & 5.3 & 5.1 & 4.4 & 3.9 & 3.6 \\
\hline TRANYCOM & 5.7 & 3.7 & 5.7 & 7.0 & 4.7 & 4.7 & 3.4 & 3.8 & 3.3 & 2.8 \\
\hline SERV & 6.1 & 6.8 & 3.5 & 7.2 & 8.1 & 4.1 & 2.9 & 8.9 & 4.0 & 4.4 \\
\hline SERVCOM & 4.3 & 3.3 & 3.0 & 5.3 & 3.8 & 2.7 & 5.2 & 3.4 & 2.6 & 3.5 \\
\hline TOTAL & 6.0 & 3.9 & 4.3 & 5.8 & 5.0 & 4.7 & 0.2 & 4.2 & 3.5 & 2.7 \\
\hline
\end{tabular}

Fuente: elaboración propia. 
Tabla 3

Resultados de las estimaciones de los modelos con datos de panel: ciclo político-económico en México, 1998-2013

\begin{tabular}{|c|c|c|c|c|c|c|c|c|c|c|}
\hline \multirow[t]{2}{*}{ Regresión } & \multicolumn{5}{|c|}{ Modelo 1} & \multicolumn{5}{|c|}{ Modelo 2} \\
\hline & Agrupada & EA & $\mathrm{EF}$ & $\mathrm{MCGF}^{\mathrm{a}}$ & $\mathrm{EECP}^{\mathrm{a}}$ & Agrupada & EA & $\mathrm{EF}$ & MCGF $^{\mathrm{a}}$ & $\mathrm{EECP}^{\mathrm{a}}$ \\
\hline Constante & $\begin{array}{l}1.4540 \\
(0.000)\end{array}$ & $\begin{array}{l}1.3988 \\
(0.007)\end{array}$ & $\begin{array}{l}1.3926 \\
(0.000)\end{array}$ & $\begin{array}{l}2.8483 \\
(0.000)\end{array}$ & $\begin{array}{l}2.7449 \\
(0.000)\end{array}$ & $\begin{array}{l}1.3749 \\
(0.000)\end{array}$ & $\begin{array}{l}1.3097 \\
(0.012)\end{array}$ & $\begin{array}{l}1.3020 \\
(0.000)\end{array}$ & $\begin{array}{l}2.8394 \\
(0.000)\end{array}$ & $\begin{array}{l}2.8524 \\
(0.000)\end{array}$ \\
\hline$E^{a}$ & $\begin{array}{l}2.0657 \\
(0.000)\end{array}$ & $\begin{array}{l}1.9046 \\
(0.000)\end{array}$ & $\begin{array}{l}1.8833 \\
(0.000)\end{array}$ & $\begin{array}{l}0.1462 \\
(0.401)\end{array}$ & $\begin{array}{l}0.1608 \\
(0.659)\end{array}$ & $\begin{array}{l}2.1760 \\
(0.000)\end{array}$ & $\begin{array}{l}2.0275 \\
(0.000)\end{array}$ & $\begin{array}{l}2.0080 \\
(0.200)\end{array}$ & $\begin{array}{l}0.0960 \\
(0.621)\end{array}$ & $\begin{array}{l}-0.0365 \\
(0.927)\end{array}$ \\
\hline$E^{p}$ & $\begin{array}{l}- \\
-\end{array}$ & $\begin{array}{l}- \\
-\end{array}$ & $\begin{array}{l}- \\
-\end{array}$ & $\begin{array}{l}- \\
-\end{array}$ & $\begin{array}{l}- \\
-\end{array}$ & $\begin{array}{l}2.8412 \\
(0.000)\end{array}$ & $\begin{array}{l}2.6962 \\
(0.000)\end{array}$ & $\begin{array}{l}2.6777 \\
(0.000)\end{array}$ & $\begin{array}{l}0.6190 \\
(0.007)\end{array}$ & $\begin{array}{l}0.6721 \\
(0.146)\end{array}$ \\
\hline$E^{d}$ & $\begin{array}{l}2.3020 \\
(0.000)\end{array}$ & $\begin{array}{l}2.2531 \\
(0.000)\end{array}$ & $\begin{array}{l}2.2474 \\
(0.000)\end{array}$ & $\begin{array}{l}0.7509 \\
(0.000)\end{array}$ & $\begin{array}{l}0.9482 \\
(0.008)\end{array}$ & $\begin{array}{l}1.2658 \\
(0.007)\end{array}$ & $\begin{array}{l}1.3531 \\
(0.002)\end{array}$ & $\begin{array}{l}1.3669 \\
(0.002)\end{array}$ & $\begin{array}{l}0.4207 \\
(0.056)\end{array}$ & $\begin{array}{l}-0.0182 \\
(0.967)\end{array}$ \\
\hline$w$ & $\begin{array}{l}-0.0571 \\
(0.293)\end{array}$ & $\begin{array}{l}-0.0792 \\
(0.148)\end{array}$ & $\begin{array}{l}-0.0828 \\
(0.134)\end{array}$ & $\begin{array}{l}-0.0381 \\
(0.311)\end{array}$ & $\begin{array}{l}-0.1772 \\
(0.010)\end{array}$ & $\begin{array}{l}-0.0601 \\
(0.282)\end{array}$ & $\begin{array}{l}-0.0864 \\
(0.128)\end{array}$ & $\begin{array}{l}-0.0908 \\
(0.114)\end{array}$ & $\begin{array}{l}-0.0442 \\
(0.274)\end{array}$ & $\begin{array}{l}-0.1583 \\
0.028\end{array}$ \\
\hline$y$ & $\begin{array}{l}0.2368 \\
(0.000)\end{array}$ & $\begin{array}{l}0.2986 \\
(0.000)\end{array}$ & $\begin{array}{l}0.3069 \\
(0.000)\end{array}$ & $\begin{array}{l}0.1652 \\
(0.000)\end{array}$ & $\begin{array}{l}0.1236 \\
(0.001)\end{array}$ & $\begin{array}{l}0.2312 \\
(0.000)\end{array}$ & $\begin{array}{l}0.2948 \\
(0.000)\end{array}$ & $\begin{array}{l}0.3035 \\
(0.000)\end{array}$ & $\begin{array}{l}0.1636 \\
(0.000)\end{array}$ & $\begin{array}{l}0.1159 \\
(0.002)\end{array}$ \\
\hline $\mathrm{R}^{2}$ & 0.1356 & 0.0842 & 0.0839 & & 0.0652 & 0.1338 & 0.0822 & 0.0818 & & 0.0598 \\
\hline $\begin{array}{l}\text { Breusch-Pagan } \\
F\end{array}$ & & 0.0000 & 0.0000 & & & & 0.0000 & 0.0000 & & \\
\hline Hausman & & 0.3746 & & & & 0.5250 & & & & \\
\hline Wooldridge $F$ & 0.0003 & 0.0003 & & & & & & & & \\
\hline
\end{tabular}

Fuente: elaboración propia.

${ }^{a}$ Modelos que presentan resultados corregidos para el modelo de efectos aleatorios. 
Errores Estándar Corregidos para Panel (EECP) a través del método sugerido por Prais y Winsten $(1954)^{20}$, que resuelve los problemas de autocorrelación y heteroscedasticidad, asumiendo un proceso autorregresivo de primer orden y utilizando el método de MCGF. Los resultados se muestran en la tabla 3.

Las estimaciones reportadas en la tabla 3 muestran que, en general, el coeficiente de determinación $\left(R^{2}\right)$ es bajo en las regresiones, con valores que fluctúan entre menos del $6 \%$ y poco más del $13 \%$. No obstante, en los 2 modelos se encuentra consistentemente un efecto positivo de la producción sobre el empleo sectorial, lo cual sustenta la hipótesis keynesiana planteada previamente de que la demanda efectiva, medida con la producción, es un determinante central del empleo, tal como han reportado otros estudios ${ }^{21}$. Por su parte, se encuentra un efecto negativo del salario sobre el empleo, lo que también es consistente con lo postulado por la nueva economía keynesiana, en particular por el modelo de los internos-externos, y lo presentado en la literatura (Escobar-Méndez, 2011). Sin embargo, aunque las estimaciones de las distintas versiones de los 2 modelos utilizados son similares en términos cualitativos (especialmente en el modelo 1), difieren de manera significativa en términos cuantitativos. Las estimaciones del coeficiente del salario de los modelos de datos agrupados, EF y EA, así como del corregido por MCGF no son estadísticamente significativas ${ }^{22}$. Sin embargo, las estimaciones corregidas por EECP son negativas y significativas en niveles cercanos al $1 \%{ }^{23}$, al igual que en Ruiz (2004) y Rodríguez y Castillo (2009), lo que podría explicarse, en el marco del modelo de la nueva economía keynesiana, por el hecho de que las variaciones del salario real no van acompañadas de cambios proporcionales en la productividad, como ha ocurrido recientemente en la economía mexicana ${ }^{24}$.

Más aun, los resultados corregidos con el procedimiento de Prais-Winsten cambian la importancia relativa de los factores tradicionales del empleo: las estimaciones muestran un coeficiente del salario superior al 0.17 en valor absoluto y por encima del 0.12 para la producción en ambos modelos, lo cual asigna una mayor importancia al primero. Estos resultados sugieren, a diferencia de estudios que se extienden hacia atrás en el tiempo, que durante los últimos 15 años se ha generado una dinámica donde el mercado va ganado terreno en la determinación del empleo. Además, dado que aquí se utiliza el salario sectorial y no el salario mínimo como variable explicatoria, los

\footnotetext{
${ }^{20}$ Esta regresión calcula los errores estándar corregidos de panel estimados suponiendo que las perturbaciones son heteroscedásticas y contemporáneamente correlacionadas a lo largo del panel. Se puede asumir que los errores están autocorrelacionados dentro del panel y que el parámetro de la autocorrelación puede ser constante entre paneles o ser diferente para cada uno. Es importante mencionar que se preserva la primera observación que en muestras pequeñas puede ser una gran ventaja.

${ }^{21}$ De hecho, diversos estudios han encontrado una relación positiva entre producción y empleo usando diferentes enfoques y muestras, tanto en la experiencia de sectores específicos como de la economía mexicana en su conjunto (véanse Mejía, 2003; Cuadra, 2008; Antón, 2011; Mejía, Rendón y Ortiz, 2014).

22 Este resultado es común en la literatura, especialmente cuando se utiliza el salario mínimo como medida de los ingresos de los trabajadores, lo que puede explicarse por su papel como ancla nominal. Véase Mejía et al. (2014) para un análisis a través de los estados.

${ }^{23}$ No obstante, las propiedades de los 2 métodos de corrección por heteroscedasticidad y autocorrelación aquí utilizados siguen siendo objeto de debate, por lo que se requiere profundizar en el análisis de estas relaciones para confirmar estos hallazgos.

${ }^{24}$ La información disponible publicada por el INEGI (www.inegi.org.mx) muestra que la productividad de los diferentes sectores de la economía nacional para diferentes subperiodos ubicados entre 2000 y 2015 ha crecido a tasas que varían entre -0.9 y $1.3 \%$ para los sectores de la construcción y del comercio al mayoreo, respectivamente, lo que contrasta con el crecimiento de los salarios, que se ha ubicado entre el 0.8 y el $5.0 \%$ en los sectores de transportes y comunicaciones y agricultura, ganadería y silvicultura, respectivamente. Aunque esta información no corresponde a los mismos sectores y periodos, permite tener una idea aproximada de cómo han evolucionado estas variables.
} 
resultados pueden estar reflejando el papel del primero como mecanismo de ajuste en el mercado laboral, conjetura que resulta razonable si tomamos en cuenta que el segundo ha tenido más bien un papel de ancla nominal en la estrategia de control de la inflación, por un lado, y que su influencia en la determinación de los demás ingresos de los trabajadores ha ido perdiendo fuerza, como Kaplan y Pérez (2006) han argumentado. Más aun, este proceso podría profundizarse a partir de la reforma laboral aprobada en México en $2012^{25}$.

En relación con la evidencia del CPE, los resultados, en cualquiera de las especificaciones estimadas, no sustentan la hipótesis planteada, ya que los coeficientes estimados no son estadísticamente significativos y en los pocos casos en que lo son, no tienen el signo esperado. Específicamente, los efectos previos a la elección (coeficiente de $\mathrm{E}^{a}$ ) nunca son estadísticamente significativos, en tanto que los efectos posteriores (coeficiente de $\mathrm{E}^{d}$ ) son significativos en las estimaciones corregidas del modelo 1, pero tienen signo positivo, opuesto al esperado. Por su parte, los coeficientes estimados son del signo correcto y estadísticamente significativos en las especificaciones de datos agrupados, EF y EA del modelo 2, propiedades que se invierten en los modelos estimados por MCGF y EECP. Además, en la estimación corregida por el método PraisWinsten, el coeficiente del periodo de transición $\left(\mathrm{E}^{p}\right)$ presenta un signo contrario al esperado que además no es estadísticamente significativo.

De estos resultados se pueden derivar algunas implicaciones interesantes. No existe evidencia de CPE en el empleo sectorial, de manera que su dinámica parece estar determinada de manera significativa por la producción (medida de demanda efectiva) y por el salario específico del sector. En otros términos, las fluctuaciones en el empleo, incluso las que pudieran ocurrir en torno a los periodos de elecciones, se pueden explicar por las fluctuaciones en la producción y en el salario real y no por la manipulación que se pueda hacer de la política económica para inducir resultados favorables a los ojos de los electores. Estos resultados difieren de otros publicados previamente, lo que se puede explicar por el hecho de que aquí se introducen variables de control que miden los efectos del ciclo económico y las condiciones de mercado en el empleo sectorial.

\section{Conclusiones}

En este trabajo se estimaron 2 modelos de panel para identificar la presencia de CPE en el comportamiento del empleo sectorial de México. Los modelos incorporan variables ficticias y de control; las primeras capturan el efecto de las elecciones presidenciales y las segundas el efecto del ciclo económico (medido por el crecimiento del PIB) y de las condiciones del mercado (medidas por el crecimiento del salario real). El impacto de las elecciones se especifica de 2 maneras distintas: en un primer modelo se distinguen sus efectos durante el año anterior a su celebración de los del año posterior (donde se incluye el trimestre de la elección), mientras que en el segundo se considera por separado el periodo que transcurre entre la elección y el cambio de gobierno, e igualmente se analiza el efecto un año antes de la elección y un año después de que se toma el poder.

Los resultados muestran efectos positivos de la producción y negativos del salario sobre el empleo, lo cual es consistente con la literatura empírica en general y con la hipótesis del modelo de los internos-externos. Una vez que se da cuenta de estos factores económicos, no se halla evidencia de CPE en el empleo sectorial de México. Aunque al parecer el empleo aumenta antes

\footnotetext{
25 Adicionalmente, Rodríguez (2005) sostiene que los salarios reales son cada vez más flexibles, lo que propicia que bajo diferentes escenarios de crecimiento económico tengan relativamente poca influencia en el empleo.
} 
y durante las elecciones y el periodo de transición, ese crecimiento no resulta estadísticamente significativo. Más aun, el periodo postelectoral se caracteriza por incrementos en el empleo, lo que contradice la hipótesis del CPE, aunque en este caso tampoco las estimaciones son significativas en los modelos corregidos.

En general, estos resultados muestran lo difícil que es lograr que las políticas «electoreras» tengan efectos reales que induzcan a los votantes a apoyar al partido en el poder. Así, aun cuando los gobernantes puedan tener la intención de manipular la política económica para beneficiarse, no siempre se logran los resultados deseados, lo cual es consistente con la evidencia reportada para otras latitudes. Sin embargo, resulta evidente la necesidad de profundizar en el análisis de las manifestaciones del CPE en indicadores alternativos de empleo y desempleo que permitan confirmar los hallazgos aquí presentados, así como en medidas de producción que identifiquen los sectores más sensibles a la manipulación política. Del mismo modo, resulta imperante analizar la presencia del CPE en función de los partidos en el poder o las diferencias regionales a lo largo del país, por citar algunos temas. Aunque varios estudios en curso van dando cuenta de estos procesos, el desperdicio económico y social que significa el uso de recursos públicos para alcanzar objetivos personales o de partido exige la profundización del análisis de estas prácticas con el fin de diseñar mecanismos que puedan evitarlas.

\section{Referencias}

Alesina, A., Roubini, N. y Cohen, G. (1999). Political Cycles and the Macroeconomy (2a imp.). Cambridge: The MIT Press.

Antón, A. (2011). Efectos del ciclo económico en Estados Unidos sobre la producción y el empleo en México. En P. Mejía y M. Morales (Eds.), Integración y recesión económica en el binomio México-Estados Unidos (pp. 143-162). Toluca: UAEM.

Aparicio J., Márquez J. (2005). Diagnóstico y especificación de modelos panel en Stata 8.0. Manual de Modelos de Panel con Stata [consultado 18 Jul 2014]. Disponible en: https://\#q=Diagn\%C3\%B3stico+y+especificaci\%C3\%B3n+ de+modelos+panel+en+Stata+8.0.+Manual+de+Modelos+de+Panel+con+Stata.+

Berger, H. y Woitek, U. (1997). Searching for political business cycles in Germany. Public Choice, 91(2), 179-197. http://dx.doi.org/10.1023/A:1004970926840

Borsani, H. (2003). Elecciones, gobiernos mayoritarios y resultados macroeconómicos en América Latina (1979-1998). Desarrollo Económico, 43(171), 389-412. http://dx.doi.org/10.2307/3455891

Breusch, T. y Pagan, A. (1980). Lagrange multiplier test and its applications to model specification in econometrics. The Review of Economic Studies, 47(1), 239-253. http://dx.doi.org/10.2307/2297111

Camacho Z. (2014). Gobierno intensifica la guerra de baja intensidad: EPR [consultado 18 Oct 2014]. Disponible en: http://contralinea.info/archivo-revista/index.php/2014/01/29/gobierno-intensifica-la-guerra-de-baja-intensidad-epr/

Cámara de Diputados del H. Congreso de la Unión (2014), Ley General de Instituciones y Procedimientos Electorales [consultado 22 Sep 2014]. Disponible en: <http://www.diputados.gob.mx/>

Centro de Estudios de las Finanzas Públicas (2014). Calendario del Presupuesto de Egresos de la Federación 2013 [consultado 22 Sep 2014]. Disponible en: <http://www.cefp.gob.mx/publicaciones/nota/2013/>

Cervantes, M., López, P. y García, P. (2014). Los ciclos politicos en México, 1960-2011. Economía, Teoría y Práctica, 40, 195-235. http://www.izt.uam.mx/economiatyp/numeros/numeros/40/articulos_PDF/40_7_Articulo.pdf

Cuadra G. (2008). Hechos estilizados del ciclo económico en México, Documento de investigación, No. 2008-14, Banco de México. http://www.banxico.org.mx/dyn/documents/\%7BA20A8FCF-2143-B36F-E7A9-509159A6AC64\%7D.pdf

Drukker, D. (2003). Testing for serial correlation in linear panel-data. The Stata Journal, 3(2), 168-177. http://www.stata-journal.com/sjpdf.html?articlenum=st0039

Escobar-Méndez, A. (2011). Determinantes del empleo en la industria manufacturera en México. Papeles de Población, 17(67), 251-276. http://www.redalyc.org/articulo.oa?id=11219005008

Flores, D. (2007). Elecciones y ciclos económicos en México. El Trimestre Económico, 74(2), 467-474. http://www.jstor. org/stable/20857121 
Gámez C. (2004). El Ciclo Político Oportunista y la Economía Mexicana (1980-2004), Ponencia, II Coloquio Predoctoral Latinoamericano Puerto Plata, Santo Domingo, XXIX Asamblea Anual de CLADEA. http://eprints.uanl.mx/ 1465/1/CLADEA_\%282004\%29.pdf

Gámez, C. (2010). El Ciclo Político y la Economía Mexicana. Monterrey: Universidad Autónoma de Nuevo León.

Gámez, C. (2012). Ciclo político, demanda agregada y formación de capital en México. Cofactor, 3(6), 44-60. http://cofactor.edomex.gob.mx/sites/cofactor.edomex.gob.mx/files/files/cofactor\%206/cofactor_pdf_cof6_art2.pdf

Gordon, R. J. (1990). Whats is New Keynesian Economics? Journal of Economic Literature, $28(3), 1115-1171$. https://www.jstor.org/stable/2727103?seq=1\#page_scan_tab_contents

Greene, W. (1998). Análisis econométrico (3. ${ }^{\mathrm{a}}$ ed.). New Jersey: Prentice Hall.

Grier, K. (1987). Presidential election and Federal Reserve policy: An empirical test. Southern Economic Journal, 54(2), 475-486. http://dx.doi.org/10.2307/1059330

Hausman, J. (1978). Specification tests in econometrics. Econometrica, 46(6), 1251-1272. http://dx.doi.org/10.2307/ 1913827

Hibbs, D. (1977). Political parties and macroeconomic policy. American Political Science Review, 71(4), $1466-1487$. http://dx.doi.org/10.1017/s0003055400269712

Im, K., Pesaran, H. y Shin, Y. (2003). Testing for unit roots in heterogeneous panels. Journal of Econometrics, 115, 53-74. http://dx.doi.org/10.1016/s0304-4076(03)00092-7

Johnston, J. y DiNardo, J. (1997). Econometrics Methods (4th ed.). California: McGraw Hill.

Kaplan, D. y Pérez, F. (2006). El efecto de los salarios mínimos en los ingresos laborales de México. El Trimestre Económico, 73(289), 139-173. http://www.redalyc.org/articulo.oa?id=31340945005

Larraín, F. y Assael, P. (1995). Cincuenta años de ciclo político económico en Chile. Cuadernos de Economía, 32(96), 129-150. http://www.jstor.org/stable/41951273?seq=1\#page_scan_tab_contents

Larraín, F. y Assael, P. (1997). El ciclo político económico en Chile en el último medio siglo. Estudios Públicos, 68, 197-214. http://www.cepchile.cl/el-ciclo-politico-economico-en-chile-en-el-ultimo-medio-siglo/cep/2016-03-03/ 183741.html

Lindbeck, A. y Snower, D. (1991). The insider-outsider theory of employment and unemployment. Journal of Economics, 93(3), 472-474. http://dx.doi.org/10.2307/3440189

Mac Rae, D. (1977). A Political model of the business cycle. Journal of Political Economy, 85, $239-263$. http://dx.doi.org/10.1086/260561

Magaloni B. (2000). Institutions, Political Opportunism and Macroeconomic Cycles: México 1970-1998, Conferencia, Instituciones Políticas y Económicas. El crecimiento en América Latina, Instituto de Historia de la Universidad de Stanford. http://www.stanford.edu/group/sshi/Conferences/1999-2000/SpringPapers/magaloni.pdf

Mahía, R. (2000). Introducción a la especificación y estimación de modelos con datos de panel [tesis doctoral]. Universidad Autónoma de Madrid. https://www.uam.es/personal_pdi/economicas/rmc/econometria/ pdf/Intro_Datos\%20de\%20panel_final.pdf

Malcomson, J. (1981). Unemployment and the efficiency wage hypothesis. Economic Journal, 91(364), 848-866. http://dx.doi.org/10.2307/2232496

Mankiw, G. y Romer, D. (1991). Introduction. New Keynesian economics. Mankiw I y II, Massachusetts: MIT Press.

Mayorga, M. y Muñoz, E. (2000). La técnica de datos de panel: una guía para su uso e interpretación. Costa Rica: Banca Central de Costa Rica, División Económica, Departamento de Investigaciones Económicas.

Mejía, P. (2003). Regularidades empíricas en los ciclos económicos de México: producción, inversión, inflación y balanza comercial. Economía Mexicana, Nueva Época, XII(2), 231-269. http://www.redalyc.org/articulo.oa?id=32312203

Mejía, P. y Erquizio, A. (2012). Expansiones y recesiones en los estados de México (1. ${ }^{\mathrm{a}}$ ed.). Hermosillo: UNISONPearson-UAEM.

Mejía, P., Rendón, L. y Ortiz, K. N. (2014). Reforma laboral y fluctuaciones cíclicas del empleo manufacturero en los estados de México. En P. Mejía y V. Torres (Eds.), Efectos de las reformas estructurales en las fluctuaciones cíclicas y el crecimiento económico en México (pp. 210-244). México: UAEM-Eón.

Milani, F. (2010). Political business cycle in the New Keynesian Model. Economic Inquiry, 48(4), 896-915. http://dx.doi.org/10.1111/j.1465-7295.2009.00212.x

Molina, I. (2001). Ciclo electoral y políticas públicas en Costa Rica (1890-1948). Revista Mexicana de Sociología, 63(3), 67-98. http://dx.doi.org/10.2307/3541242

Montero R. (2011). Efectos fijos o aleatorios: Test de especificación, Documento de Trabajo en Economía Aplicada, Universidad de Granada. http://www.ugr.es/ montero/matematicas/especificacion.pdf

Morales, D. (2007). Efectos del cambio de poder en el sector manufacturero de México. Ensayos, XXVI(2), 1-18. https://www.economia.uanl.mx/revistaensayos/xxvi/2/Efectos-del-cambio-de-poder.pdf 
Nordhaus, W. (1975). The political business cycle. Review of Economic Studies, 42(2), 169-190. http://dx.doi.org/10.2307/ 2296528

Prais S., Winsten C. (1954). Trend estimators and serial correlation, Working paper No. 383, Cowles Commission.

Remmer, K. (1993). The political economy of elections in Latin America, 1980-1991. The American Political Science Review, 87(2), 393-407. http://dx.doi.org/10.2307/2939049

Rodríguez, M. y Castillo, R. (2009). Empleo, productividad y salarios en México: un análisis de corto y de largo plazo para el sector manufacturero. EconoQuantum, 5(2), 7-21. http://dx.doi.org/10.18381/eq.v5i2.92

Rodríguez A. (2005). «Dinámica del empleo y las remuneraciones reales en México: Evolución en los últimos treinta años y perspectivas». Documento de Investigación No. 0506, Department of Economics, Universidad Iberoamericana.

Rogoff, K. (1990). Equilibrium political budget cycles. American Economic Review, 80(1), 21-36. http://scholar. harvard.edu/files/rogoff/files/51_aer90.pdf

Romer, D. (2011). Advanced Macroeconomics. Nueva York: McGraw-Hill.

Ruiz, R. (2004). Salario mínimo: consideraciones económicas y sociales para su determinación. Panorama Socioeconómico, 28, 1-13. http://www.panorama.utalca.cl/dentro/2004-may/SalarioMinimo\%5B1\%5D.pdf

SHCP (2013). Primero, segundo y tercer trimestres de la economía mexicana. Informes sobre la situación económica, las finanzas públicas y la deuda pública. Boletines de prensa. D.F. Disponible en: http://finanzaspublicas.hacienda.gob.mx/ es/Finanzas_Publicas/Informes_al_Congreso_de_la_Union; http://finanzaspublicas.hacienda.gob.mx/work/models/ Finanzas_Publicas/docs/congreso/infotrim/2013/it/01inf/itindc_201301.pdf (1.er trimestre); http://finanzaspublicas. hacienda.gob.mx/work/models/Finanzas_Publicas/docs/congreso/infotrim/2013/iit/01inf/itindc_201302.pdf (2..$^{\circ}$ trimestre); http://finanzaspublicas.hacienda.gob.mx/work/models/Finanzas_Publicas/docs/congreso/infotrim/2013/iiit/ 01inf/itindc_201303.pdf (3.er trimestre).

Villasuso J. (2000). Reformas estructurales y política económica en Costa Rica, Serie Reformas Económicas, Documento de Trabajo No. 64, Santiago de Chile, CEPAL.

Wooldridge, J. (2009). Introducción a la econometría. Un enfoque moderno (4. a ed.). México: Cengage, Learningn Latin America. 